\title{
Congestion Avoidance for Recharging Electric Vehicles Using Smoothed Particle Hydrodynamics
}

\author{
Qazi R. Hamid and Javier A. Barria, Member, IEEE
}

\begin{abstract}
In this paper, a novel approach for recharging electric vehicles (EVs) is proposed based on managing multiple discrete units of electric power flow, named energy demand particles (EDPs). Key similarities between EDPs and fluid particles (FPs) are established that allow the use of a smoothed particle hydrodynamics (SPH) method for scheduling the recharging times of EVs. It is shown, via simulation, that the scheduling procedure not only minimizes the variance of voltage drops in the secondary circuits, but it also can be used to implement a dynamic demand response and frequency control mechanism. The performance of the proposed scheduling procedure is also compared with alternative approaches recently published in the literature.
\end{abstract}

Index Terms-Electric power distribution, electric vehicles, power flow, smoothed particle hydrodynamics, voltage congestion.

\section{INTRODUCTION}

A $S$ the uptake of electric vehicles (EVs) increases, the recharging of EVs in residential electric power distribution systems will present significant operational challenges. For example, on a typical weekday, household owners will return home at roughly the same time and they will plug in their EVs. As it is known that recharging an EV can represent a non-negligible load $(10 \mathrm{~kW}$ to $30 \mathrm{~kW})$ on the distribution system, if several EVs attempt to recharge at the same time, the secondary circuits and the distribution transformer can become overloaded [1]. Under these circumstances, the voltage at customer premises might drop below acceptable and/or statutory limits. Moreover, in the case of severe overload, the distribution conductors might get damaged due to overheating.

There are at least two approaches that can be used to neutralise the impacts of such a scenario on the distribution system. 1) The distribution system operator (DSO) may upgrade the network infrastructure by installing bigger transformers and thicker conductors, but such an upgrade could be very costly. Especially, if the durations of overloaded periods are short and hence will result in underutilised assets for most of the time. 2) The DSO can install software components in the recharging sockets that will enable the coordination of EVs' recharging activities [2], [3]. In line with the second approach, this paper proposes a novel method to coordinate the recharging of EVs with explicit

Manuscript received July 03, 2014; revised October 28, 2014 and February 04, 2015; accepted March 05, 2015. Paper no. TPWRS-00911-2014.

The authors are with the Department of Electrical and Electronic Engineering, Imperial College London, London SW7 2AZ, U.K. (e-mail: j.barria@imperial. ac.uk).

Color versions of one or more of the figures in this paper are available online at http://ieeexplore.ieee.org.

Digital Object Identifier 10.1109/TPWRS.2015.2412653 consideration of the voltage drops and the demand side participation for frequency response. In comparison with random uncoordinated recharging, the method here proposed not only diminishes voltage drops but also improves both security and adequacy of electric power systems.

This paper is organized as follows: In Section II, the literature is reviewed and relevant research work is identified. In Section III, the relationship between voltage drops and active power flow is explored, and an analogy between voltage drops in the secondary circuits and the pressure in a system of fluids is presented. In Section IV, the proposed scheduler builder is presented. In Section V, the smoothed particle hydrodynamics (SPH) approach is evaluated and results are presented for a scaled down IEEE 34-bus test feeder. Final remarks are presented in Section VI.

\section{RELATED WORK}

Recently, there has been an increasing interest in investigating the impact of EV recharging schemes and the impact of their integration in the electric power system. Depending on the particular role that EVs are expected to play, researchers have pursued different lines of investigation. Several papers can be found that try to flatten the aggregate $(\mathrm{EV}+$ non-EV) demand on the electric power system [4], [5]. Other researchers have: 1) studied the use of EVs as small electric power generators to supply electric power at peak times [6], [7]; 2) investigated the impacts of uncontrolled or uncoordinated recharging of EVs on electric power system [8]-[10]; or 3) proposed algorithms for scheduling and recharging of EVs to reduce impacts on the distribution system [11], [12].

The following literature review focuses only on research efforts that consider the impact of recharging rate profiles of EVs on the mitigation of voltage congestion, and voltage congestion aware frequency control services.

Clement et al. [9], [13] appear to be the first to have suggested coordinated recharging of EVs based on consideration of voltage in the distribution system. The study in [13] is based on a stochastic simulation of EVs' energy demand requirements on a scaled down IEEE 34-bus distribution feeder that is regarded as a secondary circuit. EVs are recharged during two periods of time in a day; they are either considered connected or disconnected throughout the period, and they recharge at maximum rate. This paper presents an alternative scheduling procedure for recharging EVs and adapts a more general formulation because EVs can recharge at any time in the day, and can demand different amounts of energy in different periods of time. Also, the work presented in [13] focuses on calculating the impacts of recharging EVs using stochastic simulation of various 
scenarios, while this paper presents a scheduling method that avoids congestion in the secondary circuit.

The authors in [11] propose to schedule recharging EVs based on 1) minimizing load variance or 2) maximizing the load factor, and it is also suggested that these two optimization problems are convex optimization problems. To assume equivalence between loss minimization and the minimization of voltage drop and its associated impacts, the authors make use of remarks from [9] and [13]. The equivalence relationship between load factor and losses is based on the Buller and Woodrow formula [14]. Unfortunately, this formula is regarded as unreliable for general use in the work reported by Mikic [15]. A further problem with [11] is that it neglects the topology of the distribution system, and assumes nominal voltages at each node in the distribution system. According to [11], this assumption yields a convex optimization problem, but Taleski et al. [16] have shown that the assumption of nominal voltage at each node significantly reduces the accuracy of estimates of losses. Furthermore, it can be easily shown that maximizing the load factor does not necessarily minimize the impacts on voltage drops.

Deilami et al. [12] have proposed a real time coordinated recharging scheme to improve voltage profiles (voltage drops at each one of the nodes of a secondary circuit as a function of time) and to reduce losses. A similar approach is presented in [17]. In these studies, EVs are considered as non-elastic loads with fixed recharging rate profiles. The work in [12] can also be regarded as an admission control problem rather than a scheduling problem, because EVs that can cause voltage constraint violations are denied admission and are recharged with a delay. The rescheduling mechanism implements a priority scheme which takes into account voltage sensitivities at each bus/node in the distribution system. The work in [12] considers the full topology of the distribution system and hence avoids the approximations used in [11]. The recharging scheduling procedure propose in this paper can further improve admission control schemes such as the one suggested in [12].

Recently a packetized approach to EV charging [10] has been suggested which derives from multiple access schemes for bandwidth sharing in communication networks, and solves a distributed admission control problem. The solution is based on a probabilistic automaton that minimizes EVs participation costs. In contrast, the work presented in this paper was inspired by observing how an equilibrium is reached in a system of fluids, and focuses on building, and readjusting on-line the recharging schedule of EV energy demand requirements, with the aim of minimizing voltage congestion (voltage drops) at the secondary circuit nodes of a power distribution network. The central idea is to discretize the energy demand and map it into a particle system. The equations modelling the dynamics of the particle system can then be solved using any appropriate numerical method such as, e.g., the SPH method, which was first suggested by astrophysics researchers and has since found many other applications in engineering and sciences [18]. This paper uses the SPH method to solve a simplified form of Navier Stokes equations, as also suggested in [19].

In this paper, it is also shown that the proposed approach can be used to implement a dynamic demand response mechanism for frequency control. In the existing literature, a simple dynamic demand response mechanism is to switch off devices belonging to a certain class of loads (e.g., air conditioners and refrigerators [20]) and switch them back again at a later time. This approach can be implemented in a distributed manner but often results in the so called rebound effect [21] (or recovery peak) where the loads that try to catch up create a peak in demand when they are switched on again. Recently a desynchronization scheme has been suggested [22]. The mechanism proposed in this paper can be used as an alternative to desynchronization schemes because it also avoids the rebound effect. Note that the mechanism evaluated here (Section V-D) is not a frequency controller in the strict sense, but provides a fixed amount of reduction in load in response to a drop in frequency. That is, it re-plans the recharging schedule of connected EVs so that the charging process avoids a recovery peak, and that the voltage congestion in secondary circuits does not appear after the power system's frequency has been restored.

\section{Proposed ApproACH}

\section{A. Overview of Operation of the System}

This section describes the operation of the system that builds and executes the recharging schedules for a population of connected and active EVs.

- EVs: An EV connects to a recharging socket on arrival. When an EV is connected it is characterized by 1) its energy demand requirement $\mathrm{kWh}, 2$ ) its arrival time, and 3) its departure time. An EV has an on-board computer that can estimate these characteristics by analysing the historical journey patterns of the EV, and by keeping an estimate of the state of charge of its battery [23], [24]. The energy demand requirement, as well as the departure time can be either explicitly specified by the EVs' owner, or it could be estimated by the on-board computer. When the EV is first connected, the on-board computer reports the three characteristics to the recharging socket. Subsequently the on-board computer can send updates with respect to, e.g., changes in the EV's characteristics and/or its connectivity status.

- Recharging Sockets: A recharging socket has the capability to monitor the status of connectivity of an EV to the secondary circuit node. The recharging socket, upon receiving the characteristics of the connected $\mathrm{EV}$, relays this information to a schedule builder, and upon receiving the updated recharging schedule from the schedule builder, can execute the schedule. If the recharging socket determines that the load conditions have changed, it reports the changing conditions to the schedule builder. If the power system is working under normal operation conditions, the recharging socket is required to deliver the power flow as per schedule. However, if the power system is not operating under normal conditions, the recharging socket can stop or reduce the flow of power until normal operation conditions are restored.

- Schedule Builder The schedule builder is managed by the DSO and it knows the topology of the power distribution network (e.g., the recharging sockets locations). The schedule builder receives the energy demand requirement 


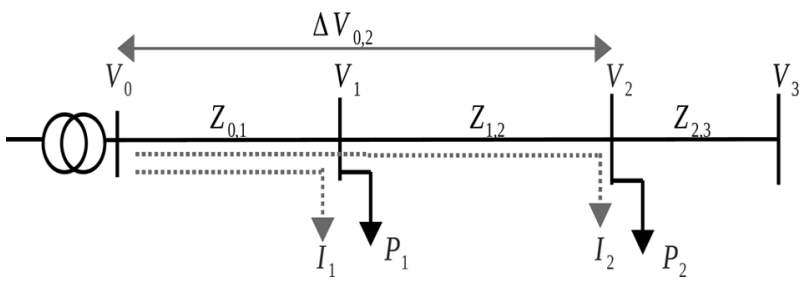

Fig. 1. Simplified diagram for a secondary circuit.

information, or the changing in load conditions from the recharging sockets, and it builds and/or readjust the recharging schedule for the whole population of active EVs. The individual EV recharging schedules are then sent to the corresponding recharging sockets. The schedule builder performs a two stage procedure. The first stage constructs an initial feasible recharging schedule. In the second stage, the initial solution is modified in pursuit of a better operational point, which considers the impact of the modified schedule on the voltage profile of the secondary circuit nodes.

1) Remarks on Communication Requirements: This paper assumes that the recharging socket is endowed with telecommunication technology that will enable a two way conversation with the EV's on board computer and the schedule builder. Similarly, the EV's on-board computer and schedule builder will be able to communicate with the recharging socket. Recharging sockets are constantly updating the schedule builder, as under normal operation conditions, new EVs can join, connect and disconnect at will. Concurrently, the recharging sockets are readjusting and executing updated recharging schedules sent by the schedule builder.

The recharging sockets are located at the secondary circuit nodes, and the schedule builder can be located near the distribution transformer. The distance between the secondary circuit nodes and the distribution transformer is typically short. Field Area Networks (FAN) can provide communication services [25]. To further assess the communication technologies is beyond the scope of this paper.

\section{B. Remarks on Voltage Drops in the Secondary Circuits and Active Power Flow}

A secondary circuit (see Fig. 1) can be very vulnerable to congestion and voltage drops due, e.g., to the unexpected appearance of new EV energy demand requirements (in particular if recharging schedules are uncoordinated). The distribution feeder typically has a tap changing transformer at the substation end and a shunt capacitor bank at a distribution feeder node. Both the tap changing transformer and the shunt capacitors can be used to regulate voltage on the distribution feeder. However, secondary circuits typically have no voltage regulating equipment and a voltage drop in a secondary circuit is reflected as a drop in utilisation voltage. Note that even though the voltages in a distribution system are affected by both active power $P$ and reactive power $Q$, when considering recharging of EVs, our interest lies in controlling active power $P$ only since a flow of reactive power $Q$ cannot be stored as energy in the batteries of EVs.

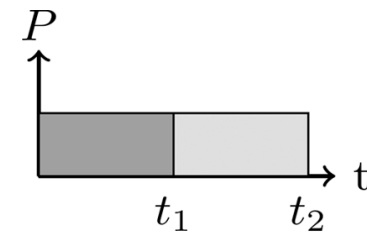

(a)

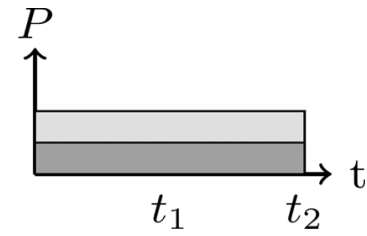

(b)
Fig. 2. Two maximum load factor recharging schedules. (a) Schedule $S_{1}$. (b) Schedule $S_{2}$

\section{1) Impact of Recharging Schedules on the Secondary Circuit} Voltages: Consider the four-node secondary circuit shown in Fig. 1, where two EVs, $E V_{1}$ and $E V_{2}$, are connected to two recharging sockets at the secondary circuit, Node 1 and Node 2 respectively. When these EVs are being recharged, they draw currents $I_{1}$ and $I_{2}$, respectively. Constant current charging is well investigated in the literature. See, e.g., [26] and [27].

In this setting, there are many possible ways in which EVs can be charged. Fig. 2(a) and (b) shows two possible ways of charging the two EVs where the different colors represent the electric power flow for $E V_{1}$ and $E V_{2}$.

This paper now proposes a novel approach for constructing recharging schedules that uses an analogy between the recharging schedule of EVs connected to different secondary circuit nodes, and a system of fluids of different densities. Let us revisit Fig. 2(a) and (b) and regard them as two virtual containers, each filled with two fluids with different densities. Assuming that the density of the dark grey fluid is higher than the density of the light grey fluid and that the two fluids are under the influence of gravity only, the two fluids will naturally settle down to $S_{2}$ (stable configuration) as $S_{1}$ (unstable configuration) will be physically impossible.

2) Analogy of Recharging Schedule to a System of Fluids: Consider the simple diagram for a secondary circuit as shown in Fig. 1. Let $\Delta V_{0,2}(t)$ be the voltage drop between bus 0 and bus 2 caused by the power flow corresponding to the recharging schedule. Then $\Delta V_{0,2}(t)$ can be written as

$$
\Delta V_{0,2}(t)=I_{1}(t) Z_{0,1}+I_{2}(t)\left(Z_{0,1}+Z_{1,2}\right) .
$$

Now consider a system of fluids with two fluids. Fluid 1 has density $\rho_{0,1}\left[\mathrm{~kg} / \mathrm{m}^{3}\right]$ and fluid 2 has density $\rho_{0,1}+\rho_{1,2}\left[\mathrm{~kg} / \mathrm{m}^{3}\right]$. Then, the expression for pressure $q\left[\mathrm{~N} / \mathrm{m}^{2}\right]$ in the system of fluids can be written as

$\left.q\left(x_{t}, y_{P}\right)\right|_{y_{P}=0}=q\left(x_{t}, 0\right)=g h_{1}\left(x_{t}\right) \rho_{0,1}+g h_{2}\left(x_{t}\right)\left(\rho_{0,1}+\rho_{1,2}\right)$

where $h_{1}\left(x_{t}\right)[\mathrm{m}]$ is the height of fluid $1, h_{2}\left(x_{t}\right)[\mathrm{m}]$ is the height of fluid $2, g\left[\mathrm{~m} / \mathrm{s}^{2}\right]$ is the acceleration of gravity, and $x_{t}[\mathrm{~m}]$ and $y_{P}[\mathrm{~m}]$ are the two coordinate axes of the system of fluids. Pictorially this analogy can be seen in Fig. 3.

Assuming that the force of gravity is normal to coordinate axis $x_{t}$, a necessary condition for equilibrium of fluid is given by (3):

$$
\frac{\partial q\left(x_{t}, 0\right)}{\partial x_{t}}=0
$$




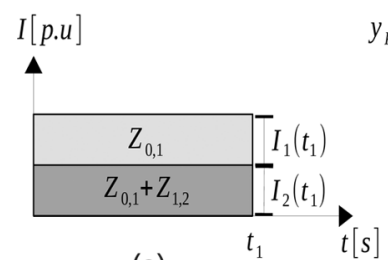

(a)

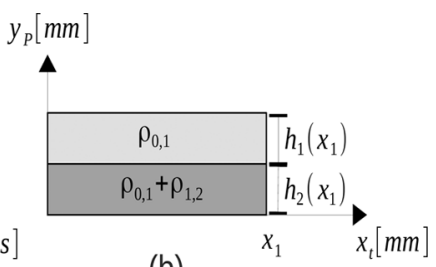

(b)
Fig. 3. Analogy of recharging schedules to a system of fluids.

The condition in (3) implies that at equilibrium the system of fluids will choose a configuration that minimizes the maximum value of pressure $q\left(x_{t}, 0\right)$ subject to 1$)$ containment of fluids within (the container) boundaries and 2) conservation of its volume.

By observing the structure of expressions in (2) and (1) and their similarity, it is our conjecture that if a recharging schedule is constructed from the equilibrium configuration of the system of fluids, then the maximum voltage drop $\left|\Delta V_{0,2}(t)\right|$ is a minimum among the maximum voltage drops caused by all other schedules.

The analogue of (3) for voltage drops in the secondary circuit is

$$
\frac{\partial\left|\Delta V_{0,2}(t)\right|}{\partial t}=0
$$

It can be verified that (4) holds for all $t$ for $S_{2}$ except for arrival and departure times of EVs, but does not hold for $S_{1}$ in Fig. 2(a) for at least one point in time $t$ which is not the arrival or departure time of either of the EVs. In fact, it can be easily verified for this charging regime (see Appendix A), that

$$
\max \left(\left|\Delta V_{0,2}(t)\right|_{2}\right) \leq \max \left(\left|\Delta V_{0,2}(t)\right|_{1}\right)
$$

where $\max \left(\left|\Delta V_{0,2}(t)\right|_{k}\right)$ is the maximum voltage drop between secondary circuit node 0 and secondary circuit node 2 for Schedule $k$.

Note from Fig. 2 and (5) these two recharging schedules have the same load factor but different voltage profiles. Hence there might exist multiple maximum load factor schedules not all of which cause minimum voltage drops. Therefore a schedule builder that builds a recharging schedule by maximizing load factor may not necessarily minimize the voltage drops.

\section{Energy Demand Particle System (EDPS) and the Fluid Particle System (FPS)}

This paper uses particle systems to 1) simulate the dynamics of a system of fluids and 2) construct a recharging schedule from the system of fluids at equilibrium. Two distinct particle systems are used namely: 1) The Energy Demand Particle System (EDPS), which contains particles that represent energy demands and 2) The Fluid Particle System (FPS), which represents a collection of particles of matter.

1) Energy Demand Particles (EDPs): An energy demand particle (EDP) is an imaginary particle that represents an active power flow of $p_{e d p} \mathrm{~kW}$ sustained over a time of $t_{e d p}[\mathrm{~h}]$ and hence delivers $p_{e d p} * t_{e d p} \mathrm{kWh}$ of power flow. Energy demand particles are used by the recharging sockets to deliver the energy demand required by the EV's batteries. Therefore EDPs acts as a load of $p_{e d p} \mathrm{~kW}$ at a secondary circuit node.

EDPs belong in a $(t, P)$ plane, where $t$ represents time and $P \mathrm{~kW}$ represents electric power. The $t$-coordinate of an EDP in the $(t, P)$ plane denotes the planned time (called the "activation time" of the EDP) at which the recharging socket will start delivering $p_{e d p} \mathrm{~kW}$ of electric power to the battery of the EV. When a recharging socket has started delivering electric power corresponding to an EDP, and has not finished yet (because, under normal operation conditions, electric power flow must be sustained for a time $t_{e d p}$ ), one can say that the EDP is "active" at the recharging socket. Several EDPs may be active at a recharging socket at a given point in time. Each EDP is related to a single secondary circuit node in the distribution system. When an EDP becomes active, a load of $p_{e d p} \mathrm{~kW}$ is added to the corresponding secondary circuit node. The aggregate electric power demand of all active EDPs at a recharging socket becomes the recharging rate profile of the EV connected to that recharging socket.

If the power system is operating under abnormal conditions, the recharging socket can stop the power flow until normal operation conditions are reestablished. For example, if a recharging socket activates an EDP, but subsequently detects a significant drop in frequency, it can react by either deferring (delaying) parts of the power flow (see example 3 in Section V-D) or by deactivating EDPs, in accordance with a pre-established operations policy. If an EDP has not been activated and the activation time has elapsed, it is removed from the current schedule, and the schedule builder is informed (so that the removed EDP can be scheduled for some other time in the future).

2) Fluid Particles (FPs): A fluid particle (FP) represents, in the context of this paper, a very small volume of fluid. An FP has a mass which is a function of the density of fluid that FP represents. For each EDP in the $(t, P)$ plane, there is a corresponding FP in the $\left(x_{t}, y_{p}\right)$ plane. The type and characteristics of the FP corresponding to a given EDP depend on the secondary circuit node to which the EDP is associated.

An FPS is a collection of FPs in which FPs can move in space. For all studied cases, initial velocity and acceleration of the FP are set to zero. That is $a(\tau=0)=0$, where $\tau$ is the simulation time in the FPS.

\section{Proposed Schedule Builder}

The proposed schedule builder constructs a recharging schedule for a population of EVs connected to a secondary circuit node in two stages. Stage 1 produces an initial recharging schedule that can be accomplished using any procedure, e.g., using a first come first served recharging policy or a maximum load factor schedule as proposed in [11]. Stage 2 [the proposed smooth particle hydrodynamics (SPH) recharging procedure] will then aim at continuously finding a better schedule, considering the impact of the modified recharging schedule on the voltages profile (i.e., reducing the voltage drops at secondary circuits nodes over time). With the help of Fig. 4, the steps followed by the proposed procedure are now further described. 


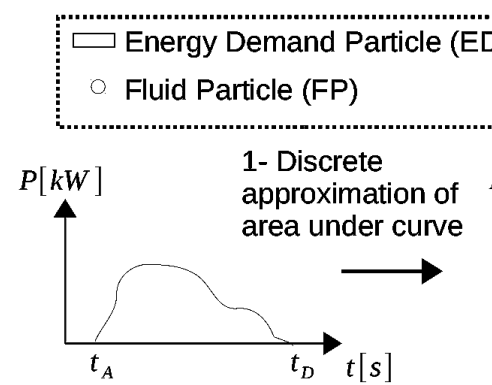

(a) Initial recharging rate profile

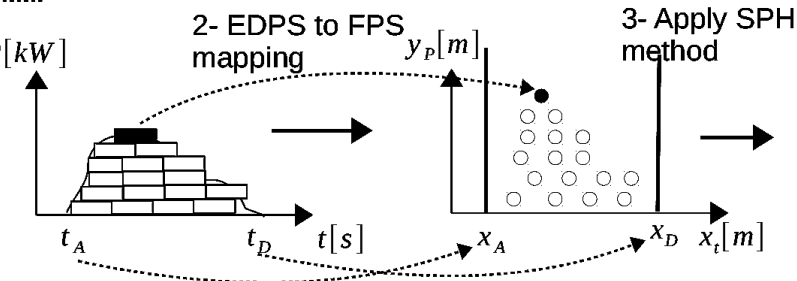

(b) construct EDPS (c) map EDPS to FPS

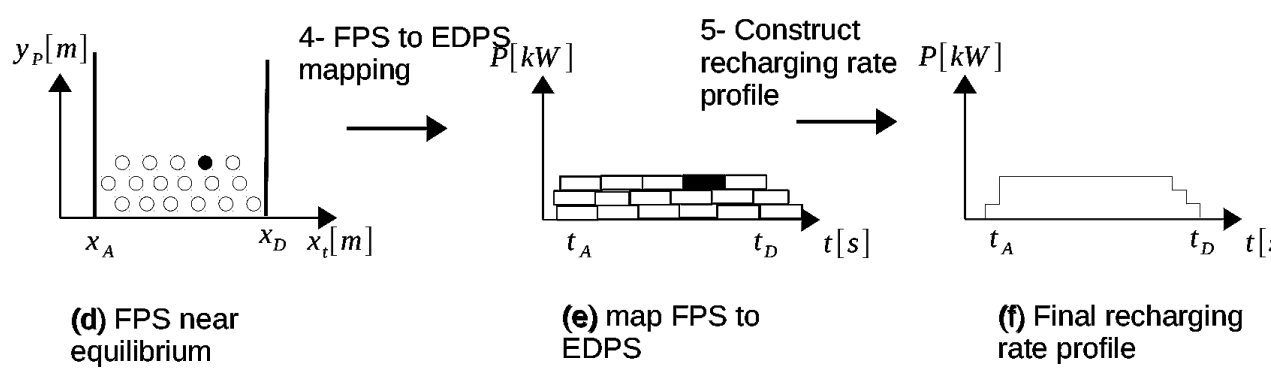

Fig. 4. Proposed schedule builder. (a) Initial recharging rate profile. (b) Construct EDPS. (c) Map EDPS to FPS. (d) FPS near equilibrium. (e) Map FPS to EDPS. (f) Final recharging rate profile.

\section{A. Step 1: Building an EDPS From an Initial Recharging Schedule}

Once the EVs' energy demand requirements are known to the schedule builder, an initial EDPS is constructed [Fig. 4(a)]. Note that the area under the curve of the recharging rate profile in Fig. 4(a) is approximated by the aggregate area of a collection of EDPs in Fig. 4(b). Thus, the power flow delivered by the recharging rate profile and the power flow delivered by the ensemble (array) of EDPs are equal, up to the error of approximation. The error of approximation is the difference between the area under a recharging rate profile and the aggregate areas of all EDPs for the recharging rate profile. When $N$ EDPs are used to approximate the area under recharging rate profile, then the error of approximation can be expressed as

$$
e=\int_{t_{A}}^{t_{D}} P(t) d t-N p_{e d p} * t_{e d p}
$$

By choosing the size of $p_{e d p}$, this error can be made arbitrary small.

\section{B. Step 2: Mapping the EDPS to an FPS}

The constructed EDPS is then mapped into an FPS as shown in Fig. 4(c). Each individual EDP in the EDPS is then mapped into a unique FP in the FPS. Given that an EDP in the $\left(t_{1}, P_{1}\right)$ plane is related to a secondary circuit node $j$ and $\mathrm{EV} k$, the FP is constructed in the $\left(x_{t 1}, y_{p 1}\right)$ plane as follows:

$$
\begin{aligned}
& x_{t 1}=K_{t, x} t_{1} \\
& y_{p 1}=K_{p, y} P_{1}
\end{aligned}
$$

where $K_{t, x}$ and $K_{p, y}$ are constants that are used to transform a point in the $(t, P)$ plane into a point in the $\left(x_{t}, y_{p}\right)$ plane. The mass assigned to an FP is calculated using (9)

$$
m=K_{0, m}+K_{Z, m}\left|Z T_{j, 0}\right|
$$

where $K_{0, m}$ and $K_{Z, m}$ are constants, and $Z T_{j, 0}$ is the sum of all impedance between secondary circuit node $j$ and the distribution transformer.

\section{Step 3: Applying the SPH Method to FPS}

Once the FPS is obtained, the SPH procedure can then be applied to it. The SPH procedure is essentially an indefinite loop which has a fixed point at the equilibrium of FPS (the SPH Procedure is described in Section IV-F1). Note that as soon as Step 3 is active and running (continuously modelling fluids flowing in a container), the mapping of the FPS to the modified EDPS (Step 4 to follow) can be performed.

\section{Step 4: Mapping FPS to EDPS}

While the SPH procedure is continuously running on the FPS, the schedule builder can map the FPS to the EDPS at any point in time, as shown in Fig. 4(e). This mapping is the inverse of the mapping used in Step 2.

\section{E. Step 5: Building the Final Recharging Schedule From EDPS}

Given an EDPS, this step constructs a recharging schedule by using the inverse of Step 1 as shown in Fig. 4(f). Although this step is called "building the final recharging schedule", the recharging schedule is not final in the sense that it cannot be changed. In fact, at any time the schedule builder can be made aware of an updated/modified FPS that is being solved by the SPH procedure (running in the background in Step 3), and from this notification the schedule builder can construct an updated recharging schedule (performing steps 4 and 5).

1) Schedule Builder Online Operation: The schedule builder is capable of dealing with unexpected perturbations like, e.g., new arrivals of EVs and sudden loss of power generation. For 
example, if a new EV connects to a recharging socket, of which the schedule builder had no prior information, the recharging socket will inform the schedule builder. The schedule builder will construct EDPs for the EV as in Step 1, map EDPs to FPs as in Step 2, and add newly mapped FPs to the already existing FPS. Thus, the SPH procedure which is continuously running in the background (Step 3) will face an augmented FPS, and the FPS will naturally pursue a new equilibrium. The new recharging schedule is then constructed using steps 4 and 5 .

2) Recharging Socket/Schedule Builder Information Exchange: The schedule builder maintains at all times the full array of EDPs representing the population of all connected EVs, and it can add or remove EDPs from the array. In contrast, the recharging socket keeps at all times the EDPs for only one EV representing the locally connected EVs. The recharging socket also informs the schedule builder of any requested changes made by the EV's on-board computer or any change in its connectivity status. The recharging socket is able to react to local constraints like, e.g., EV's maximum recharging rate limits, and earlier than expected departure (disconnection) of an EV. The schedule builder is constantly modifying the array of EDPs to represent, e.g., changes in EV's status, and updates from recharging socket on new energy demand requests.

3) Transformation of Constraints on the Energy Demand to Constraints on EDPs and FPs: There are two constraints on the demand of energy from an EV that need to be considered.

The first constraint ensures that the power flow delivered to the $E V_{j, k}$ is equal to the energy demand required by the $E V_{j, k}$ :

$$
\int_{t_{-} A_{j, k}}^{t_{-} D_{j, k}} p_{j, k}(t) d t=E D_{j, k}
$$

where $t_{-} A_{j, k}$ is the arrival time of $E V_{j, k}$ at the recharging socket, $t_{-} D_{j, k}$ is its departure time from the recharging socket, and $E D_{j, k}$ is the energy demand requirement specified by $E V_{j, k}$.

The second constraint ensures that electric power is scheduled only in a window of time for which EV is connected to a recharging socket:

$$
p_{j, k}(t)= \begin{cases}0 & \text { if } t<t_{-} A_{j, k} \\ \geq 0 & \text { if } t_{-} A_{j, k}<t<t_{-} D_{j, k} \\ 0 & \text { if } t_{-} D_{j, k}<t\end{cases}
$$

These two constraints on power flow, namely (11) and (10), can be translated into two properties of particle systems using the following remarks: 1) The EDPs associated with $E V_{j, k}$ should not move outside the boundaries defined by $t_{-} A_{j, k}$ and $t_{-} D_{j, k}$. Thus the FPs associated to these EDPs should not move outside the boundaries $x_{-} A_{j, k}$ and $x_{-} D_{j, k}$ that are obtained by transforming $t_{-} A_{j, k}$ and $t_{-} D_{j, k}$ using (7). 2) The EDPs associated with $E V_{j, k}$ are constructed in such a way that their aggregate area in the $(t, P)$ plane equals the $E D_{j, k}$. As each EDP has an area that does not change (an EDP could be removed temporary at one location, but it will have to be added back to the EDPS at a different location), the aggregate area of the EDPs will remain constant.

\section{F. Smoothed Particle Hydrodynamics (SPH) Procedure}

This section describes the Smoothed Particle Hydrodynamics $(\mathrm{SPH})$ procedure that is used in Step 3 in Section IV-C.

1) SPH Procedure: The SPH procedure applied to the FPS is here described using the pseudo code in Procedure 1. The following notation is used to describe the properties of the FPs and FPS, where a subscript $i$ appearing in the pseudo code in Procedure 1 represents the $i$ th FP:

- $m=$ mass of an FP;

- $\mathbf{x}=\left[x_{t}, y_{p}\right]^{T}=$ the position of FP in FPS;

- $\mathbf{x} \cdot x_{t}=x_{t}$ coordinate of $\mathbf{x}$;

- $\mathbf{v}=\left[v_{x}, v_{y}\right]^{T}=$ velocity of FP;

- $\mathbf{a}=\left[a_{x}, a_{y}\right]^{T}=$ net acceleration of FP;

- $\mathbf{a}_{i, q}=$ acceleration of FP $i$ due to the force generated by pressure gradient;

- $\mathbf{a}_{i, e}=$ the acceleration of FP $i$ due to the external force field, which typically is the force of gravitation;

- $\rho=$ density of FPS;

- $\rho_{r}=$ rest density of FPS;

- $q=$ pressure in the FPS;

- $\tau=$ FPS simulation time;

- $\Delta \tau=$ a time step in FPS simulation time;

- $x_{l}=$ minimum value of $x_{t}$ coordinate of an FP defines the left boundary that the FP cannot cross;

- $x_{r}=$ maximum value of $x_{t}$ coordinate of an FP defines the right boundary that the FP cannot cross;

- $K$ and $L$ are constants, which are non-negative.

Procedure 1 Pseudo code for the SPH procedure

loop $\tau \leftarrow \tau+\Delta \tau$

for each FP $i$ in the FPS do

//find current neighbors

$\mathcal{N}_{i}=\left\{j \mid\left\|\mathbf{x}_{\mathbf{j}}-\mathbf{x}_{\mathbf{i}}\right\| \leq h\right\}$

//calculate density at FP $i$ 's position

$\rho_{i} \leftarrow \sum_{j \in \mathcal{N}_{i}} m_{j} w_{\text {poly } 6}\left(\mathbf{x}_{\mathbf{j}}-\mathbf{x}_{\mathbf{i}}, h\right)$

//calculate pressure at FP $i$ 's position

$q_{i} \leftarrow K\left(\rho-\rho_{r}\right)$

\section{end for}

for each FP $i$ in the FPS do

//calculate acceleration of FP due to pressure //acceleration of FP is calculated directly without calculating force

$$
\begin{aligned}
& \mathbf{a}_{i, q} \leftarrow \\
& -\sum_{j \in \mathcal{N}_{i}}\left(\frac{q_{i}}{\rho_{i}^{2}}+\frac{q_{j}}{\rho_{j}^{2}}\right) w_{s p i k y}\left(\mathbf{x}_{\mathbf{j}}-\mathbf{x}_{\mathbf{i}}, h\right) \frac{\mathbf{x}_{\mathbf{j}}-\mathbf{x}_{\mathbf{i}}}{\left\|\mathbf{x}_{\mathbf{j}}-\mathbf{x}_{\mathbf{i}}\right\|}
\end{aligned}
$$

//calculate acceleration of FP due to external force field (gravitational force)

$\mathbf{a}_{i, e} \leftarrow[0,-L]^{T}$

//calculate net acceleration of FP $i$

end for $\mathbf{a}_{i} \leftarrow \mathbf{a}_{i, q}+\mathbf{a}_{i, e}$ 
for each FP $i$ in the FPS do

//calculate velocity of particle $i$ using Euler integration method.

$\mathbf{v}_{i} \leftarrow \mathbf{v}_{i}+\mathbf{a}_{i} \Delta \tau$

//calculate position of particle $i$

$\mathbf{x}_{i} \leftarrow \mathbf{x}_{i}+\mathbf{v}_{i} \Delta \tau$

//check boundaries for this FP. If FP has moved outside its allowed boundaries, assign it boundary a position.

$$
\text { if } \begin{array}{r}
\mathbf{x}_{\mathbf{i}} \cdot x_{t}<x_{l, i} \text { then } \\
\mathbf{x}_{\mathbf{i}} \cdot x_{t} \leftarrow x_{l, i}
\end{array}
$$

end if

$$
\begin{aligned}
& \text { if } \mathbf{x}_{\mathbf{i}} \cdot x_{t}>x_{r, i} \text { then } \\
& \quad \mathbf{x}_{\mathbf{i}} \cdot x_{t} \leftarrow x_{r, i} \\
& \text { end if } \\
& \text { end for } \\
& \text { end loop }
\end{aligned}
$$

Two kernels are used in this paper. The kernel $w_{\text {poly } 6}(\mathbf{r}, h)$ (12) suggested by Müller, Charypar, and Gross [19] is used due to its reduced computational cost:

$$
w_{\text {poly } 6}(\mathbf{r}, h)= \begin{cases}\frac{4}{\pi h^{8}}\left(h^{2}-r^{2}\right)^{3} & \text { if } 0 \leq r \leq h \\ 0 & \text { otherwise. }\end{cases}
$$

However, this kernel can cause particles to form clusters if it is used to compute the pressure gradient because its gradient approaches 0 when $r$ approaches 0 . Therefore, the following kernel $w_{\text {spiky }}(13)$ is used to compute pressure gradient [19]:

$$
w_{\text {spiky }}(\mathbf{r}, h)= \begin{cases}\frac{10}{\pi h^{5}}(h-r)^{3} & \text { if } 0 \leq r \leq h \\ 0 & \text { otherwise }\end{cases}
$$

where $\mathbf{r}=\left[r_{1}, r_{2}\right]^{T} \in \mathbf{R}^{2}$ and $r=\sqrt{r_{1}^{2}+r_{2}^{2}}$.

\section{RESUlts}

For the results presented in this section, a four-node secondary circuit shown in Fig. 1(a) is used, where $Z_{0,1}=Z_{1,2}=$ $Z_{2.3}=0.05+j 0.05$ p.u., $V_{s}=1 \angle 0$ p.u., $V_{\text {base }}=415 \mathrm{~V}$, and $S_{\text {base }}=1$ MVA. There are three active EVs and EV $i$, for $i=1,2,3$ is connected to a recharging socket in node $i$ of the secondary circuit.

The EDPs are constructed using $p_{e d p}=1 \mathrm{~kW}$ and $t_{e d p}=$ $300 \mathrm{~s}$, and the FPs are constructed using $K_{t, x}=12 \mathrm{~mm} / \mathrm{h}$ (each hour in time maps to $12 \mathrm{~mm}$ in the system of fluids), $y_{P 1}=$ $1 \mathrm{~mm} / \mathrm{kW}, K_{0, m}=0.5 \mathrm{mg}$, and $K_{Z, m}=0.707 \mathrm{mg} /$ p.u.

In the SPH Procedure 1 the values of parameters used are: $h=2[\mathrm{~mm}], \rho_{r}=0.2\left[\mathrm{mg} / \mathrm{mm}^{3}\right], \Delta \tau=0.1[\mathrm{~s}], K=$ $1\left[\mathrm{~mm}^{2} / \mathrm{s}^{2}\right]$, and $L=0.2 \times m_{i}\left[\mathrm{~mm} / \mathrm{s}^{2}\right]$ where $m_{i}$ is the mass of an FP for which $L$ is to be used in the SPH Procedure 1 .

\section{A. Performance Metric}

In previously published literature [11]-[13], a metric to quantitatively compare the performance of recharging schedules was not identified. When a recharging schedule is executed, it produces voltage drops that vary in time as EVs connect, wait while connected, recharge, remain idle after being fully recharged, and disconnect to start a new journey. For a two node secondary circuit, the metric (14) maps a voltage drop over time to a scalar:

$$
J_{1}=\left(\int_{0}^{T}\left(\left|V_{0}\right|-\left|V_{1}\right|\right)^{M} d t\right)
$$

where $T$ is the total time period for which a recharging schedule is constructed, $V_{0}$ is the voltage at the output of distribution transformer, $V_{1}$ is the voltage at the secondary circuit node 1 , and $M$ is a positive integer greater than one. The metric (14) implicitly attaches an exponentially increasing penalty to linearly increasing voltage drop. Note also that for a given amount of energy transfer between node 0 and node 1 , this metric yields a minimum value if energy is delivered at a constant rate, and the magnitude of the voltage drops are kept to a minimum and are constant over the entire energy transfer time.

For a multiple node secondary circuit, the metric (15) is used in this paper due to the fact that voltage drops on nodes belonging to the same secondary circuit are correlated. For example, consider a scenario where two EVs $\left(E V_{1}\right.$ and $\left.E V_{2}\right)$ are connected to node 1 and node 2, respectively. Cases of interest will arise only if recharging $E V_{1}$ causes a voltage drop at node 2 and/or recharging $E V_{2}$ causes a voltage drop at node 1. Hence, since the two voltage drops are correlated, the highest voltage drop in the shared secondary circuit is of interest and representative of the worst affected node. Note that if EVs are attached to different secondary circuits, their recharging schedule can be treated as two recharging schedules being executed in parallel:

$$
J=\int_{0}^{T}\left(10 \max _{i}\left(\left|V_{0}\right|-\left|V_{i}\right|\right)\right)^{6} d t .
$$

Since the voltage magnitudes specified in this paper are in per unit values, a scalar multiplication by 10 in (15) ensures that a voltage drop of 0.1 p.u. (10\%) is mapped to the value 1 . Metric (15) provides just one way to compare recharging schedules and future researchers might explore alternative metrics.

\section{B. Example 1: Using Admission Control Based Schedule as Initial Schedule}

The initial recharging schedule $S_{1}$ in this example is based on an admission control scheme as suggested in [12]. This scheme attempts to recharge each $\mathrm{EV}$ as soon as possible after the EV connects to a recharging socket. All three EVs connect to their respective recharging sockets at $t=0$. EV2 needs to depart after $2 \mathrm{~h}$ and 45 min (i.e., time slot 33). EV1 and EV3 will remain connected for approximately $3 \mathrm{~h}$ and $20 \mathrm{~min}$ (i.e., 40 time slots).

In Fig. 5, the recharging rate profiles are shown for the two recharging schedules, $S_{1}$ and $S_{2}$. Stage 1 starts recharging all EVs at time slots 0 and finishes recharging at time slot 15 (in this example it is possible to recharge at maximum rate simultaneously). In contrast, the final recharging schedule $S_{2}$ recharges EVs simultaneously but at a reduced recharging rate, and takes into account the fact that EV 2 departs after time slot 33. It can be noted that near time slot 33 , the recharging rate accelerates 


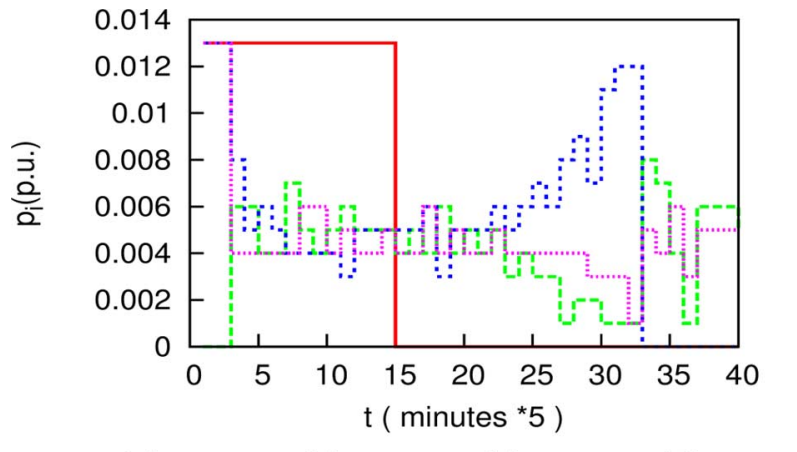

$\begin{array}{llll}\text { (a) }- & \text { (b) }-\cdots-- & \text { (c) } \cdots \cdots \cdots & \text { (d) }\end{array}$

Fig. 5. (a) $p_{1}(t), p_{2}(t), p_{3}(t)$ for initial schedule $S_{1}$, (b) $p_{1}(t)$, (c) $p_{2}(t)$, and (d) $p_{3}(t)$ for the final recharging schedule $S_{2}$.

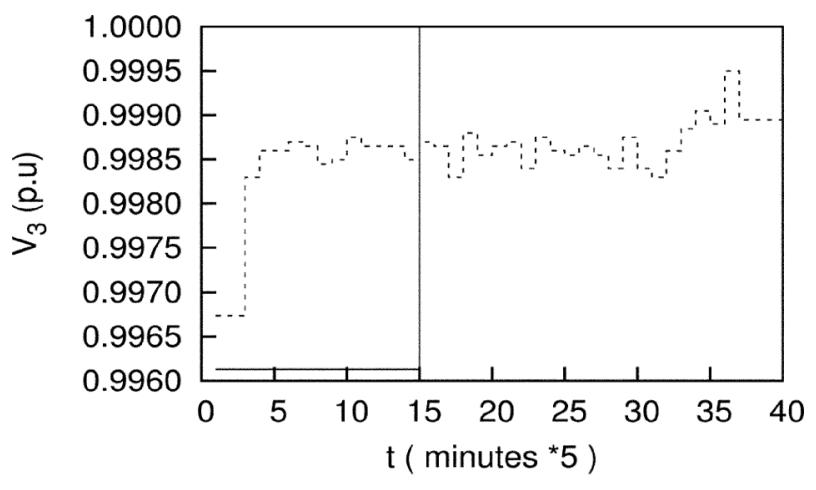

(a)

(b) $\cdots$

Fig. 6. Voltage profile: $\left|V_{3}(t)\right|$ for (a) the initial recharging schedule $S_{1}$ and (b) the final recharging schedule $S_{2}$.

TABLE I

Mean and Standard DeViation of Voltage Drop

\begin{tabular}{cllllll}
\hline Node & \multicolumn{2}{c}{$\mathbf{1}$} & \multicolumn{2}{c}{$\mathbf{2}$} & \multicolumn{2}{c}{$\mathbf{3}$} \\
& $S_{1}$ & $S_{2}$ & $S_{1}$ & $S_{2}$ & $S_{1}$ & $S_{2}$ \\
\hline$\mu\left(\left|\Delta V_{0,1}\right|\right)$ & 0.00066 & 0.00069 & 0.00112 & 0.00119 & 0.00135 & 0.00142 \\
$\sigma\left(\left|\Delta V_{0,1}\right|\right)$ & 0.00092 & 0.00018 & 0.00155 & 0.00040 & 0.00186 & 0.00048 \\
\hline
\end{tabular}

for EV2, and that for EV1 and EV3 is reduced. This effect occurs because EV2 is departing after time slot 33 . In the FPS, the FPs representing EV2 will have a right (container) boundary and cannot move to the right-hand side of time slot 33 . As a consequence, FPs for EV2 might start accumulating near the time slot, as shown in the figure. In contrast, the right (container) boundary for EV2 and EV3 FPs will become a constraint for moving particles only after time slot 40 , and hence EV2 and EV3 FPs can relocate themselves between time slots 33 and 40 .

In Fig. 6, the voltages at secondary circuit node 3 for the initial and the final recharging schedules are shown. The initial recharging schedule causes $\left|V_{3}\right|=0.9961$ p.u. (a $0.4 \%$ voltage drop in the secondary circuit) in time slots 1 to 15 and then it causes no voltage drops in time slots 15 to 40 . In contrast, the final recharging schedule $S_{2}$ causes an almost uniform $\left|V_{3}\right| \approx$ 0.9985 p.u. (approximately $0.15 \%$ voltage drop in secondary circuit) in almost all time slots.

Table I shows the mean and standard deviation of voltage drops at all three secondary circuit nodes. Here too, the mean

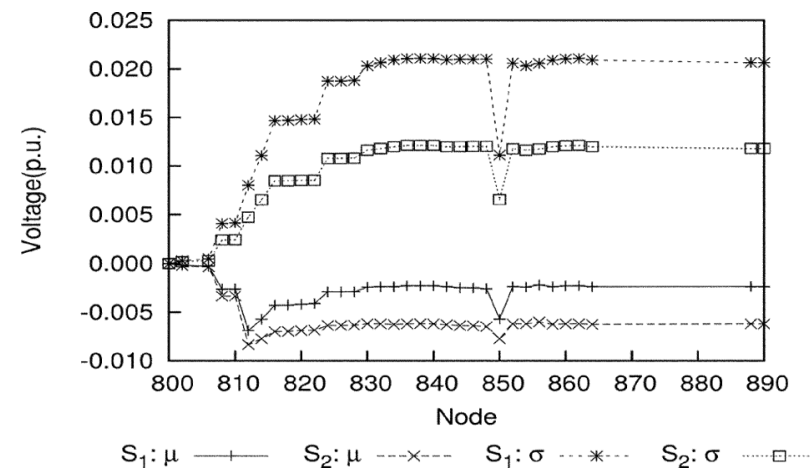

Fig. 7. Mean and standard deviation of voltage drops between distribution transformer and node $\mathrm{N}$ for $S_{1}$ and $S_{2}$.

of voltage drops is similar for both schedules, with the final schedule $S_{2}$ having slightly larger mean voltage drops, but significantly lower standard deviation of voltage drops (when compared with the initial schedule $S_{1}$ ).

Evaluating the performance metric in (15) obtains a value of $J=3.72 \times 10^{-7}$ for the initial schedule $S_{1}$, and of $J=$ $2.16 \times 10^{-8}$ for the final schedule $S_{2}$, which is further evidence that the final recharging schedule improves on the initial one.

\section{Example 2: IEEE 34-Bus Test Feeder Results}

In this example, a scaled down IEEE 34-bus feeder is considered. The lengths of the feeder sections in the IEEE 34-bus feeder have been scaled down by a factor of 100 as suggested in [13]. Exponentially distributed random numbers with a mean of 100 are generated, and these values are used as the arrival times (in minutes) of the $34 \mathrm{EVs}$. For the connected EVs uniformly distributed random numbers are generated in the interval $[30,300] \mathrm{m}$ to represent their sojourn time. The energy demand requirements from the EVs are assumed to be uniformly distributed between $3 \mathrm{kWh}$ and $10 \mathrm{kWh}$. In this example, the arrival time of the EVs is known in advance.

Two recharging schedules $S_{1}$ and $S_{2}$ are compared in Fig. 7 in terms of mean and standard deviation of voltage profiles (voltage drops) between the distribution transformer at node 800 and each node $N$ over the duration of the recharging time. In the first schedule $S_{1}$, uncoordinated recharging is assumed. Here EVs are recharged at maximum recharging rate as soon as they get connected to a recharging socket. The second recharging schedule $S_{2}$ is obtained by taking $S_{1}$ as initial schedule and using the process described in Section IV. The voltage drops are calculated for both schedules by solving a sequence of load flow problems with the recharging load of EVs, and according to schedule $S_{1}$ and $S_{2}$, respectively.

Fig. 7 shows that the proposed approach can improve the recharging schedule by reducing the standard deviation of voltage drops and hence avoiding extreme voltage drops. In this case, $S_{2}$ also decreases the mean voltage drops as compared to $S_{1}$.

Note that the node numbers in the IEEE 34-bus feeder are not ordered based on their physical proximity to the substation. This causes node 850 to experience much less voltage drops when compared to its neighbors in numeric order. 


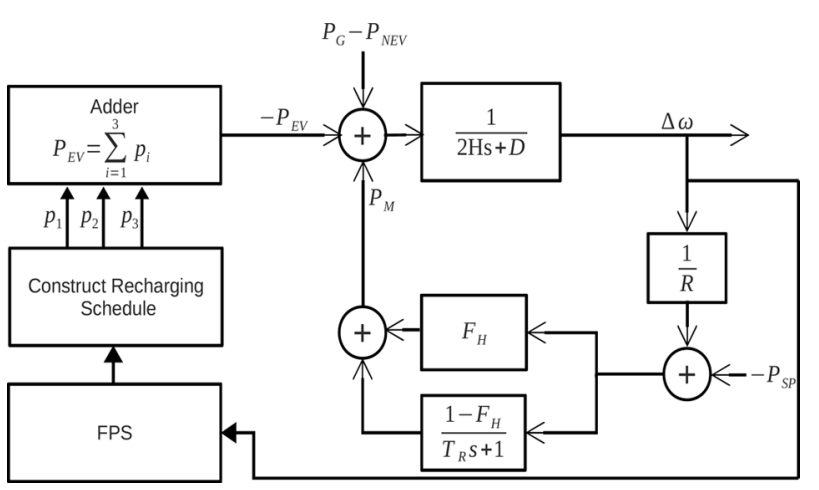

Fig. 8. Particle system and its relation to the frequency control service.

\section{Example 3: Voltage Congestion Aware Frequency Control Service}

This example highlights an additional benefit when using the proposed approach in providing a dynamic demand response to minimize the impact of a recovery peak. In the FPS, the schedule builder can dynamically shift the left boundary of the container of fluids [which is labeled as $x_{A}$ in Fig. 4(c)] towards the right, and as a consequence, defer the recharging of EVs. In this example, this dynamic shift is used to reduce the current load as a response to a sudden drop in frequency (e.g., as a consequence of a sudden failure of a large generator). An individual EV is a very small load that cannot affect the frequency of the power system. However, when a large population of EVs is recharging, deferring their recharging can have a perceptible effect on the frequency. In this example, the boundary of the FPS is shifted such that the EDPs that are scheduled to be activated in the next $10 \mathrm{~m}$ are deferred (it is assumed that secondary generators can be started within $10 \mathrm{~m}$ ). As a consequence of the shift on the boundary, the FPS is perturbed and pursues a new equilibrium.

To calculate the impact on the voltage, the following parameter values are used: $Z_{0,1}=Z_{1,2}=Z_{2,3}=0.05+j 0.05$ and $V_{0}=1 \angle 0$. There are three EVs and EV $i$ is connected to the secondary circuit node $i$ for $i=1,2,3$. The test system used for the frequency control is shown in Fig. 8, which is a scaled down electric power system where a loss of a $100-\mathrm{kW}$ generator can affect the frequency. Fig. 8 represents a reduced order frequency response model, in which the system generation is composed primarily of steam turbine units to produce mechanical power. The model captures the speed deviations as a function of acceleration power, and represents a reheat steam turbine and a speed governor. Also each EDP represents a load of $2 \mathrm{~kW}$.

The test system in Fig. 8 has the following parameter values: the inertia constant $H=3.5$; the damping factor $D=1$; the speed droop or regulation $R=0.05$; the high pressure power fraction $F_{h}=0.3$; the reheat time constant $T_{R}=8$; and the incremental power set point $P_{S P}=0$. Here $P_{\text {base }}=1 M V A$. In the initial schedule, the total instantaneous EV load is $48 \mathrm{~kW}$ on average which is 0.048 p.u. with respect to $P_{b a s e}$. Models of power systems for frequency control are discussed in [28] and interested reader is referred to [28] where the model used in this example is described in detail.

The primary frequency regulation is mainly provided by a generator driven by a steam reheat turbine, which is fitted with

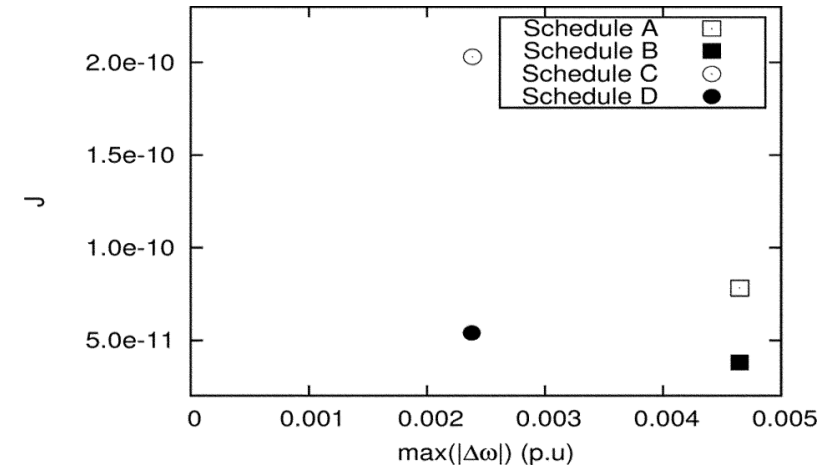

Fig. 9. $\max (|\Delta \omega|)$ as maintained by primary frequency control before restoration of frequency versus $J$ for the four schedules.

a speed governor with drop of $5 \%$. At $t=0 \mathrm{~s}$, an electric power generator of size $100 \mathrm{~kW}$ or 0.1 p.u. fails suddenly causing a $10 \%$ loss in the electric power generation. A secondary generator starts producing $100 \mathrm{~kW}$, at $t=10 \mathrm{~m}$, to compensate for the earlier loss in the electric power generation.

Four recharging schedules are compared in terms of the performance metric $J$ and their impact on the frequency response of electric power system.

1) Schedule A: This schedule is a maximum load factor schedule [11] where EVs are recharged in a sequence. The schedule is never changed during execution and hence it does not respond to a drop in frequency.

2) Schedule B: This schedule takes Schedule A as an initial schedule, and in Stage 2 a schedule is constructed from the equilibrium configuration of FPs in the FPS. Schedule B is never changed during its execution and hence it does not respond to a drop in frequency.

3) Schedule C: This schedule differs from schedule B in that it responds to a drop in frequency by simply delaying the demand for 10 minutes. Any demand scheduled at times later than 10 minutes after the drop in frequency is not affected.

4) Schedule D: This schedule takes Schedule B and uses the same FPS as used by Schedule B, but it further responds to a drop in frequency by shifting the boundary of the FPS and allowing the FPS to reach a new equilibrium, from which a new schedule is constructed and executed.

Fig. 9 shows the comparative performance of the four selected schedules, in terms of $J$ in (15).

It can be seen that Schedule B achieves a better performance when compared to Schedule A in terms of voltage impacts but is otherwise equivalent to Schedule $\mathrm{A}$ in terms of frequency response. Schedule $\mathrm{C}$ improves the frequency response but increases voltage drops and can potentially cause congestion. In this example it does not cause congestion because there are only $3 \mathrm{EVs}$ in the secondary circuit. Schedule D manages to improve the frequency response as well as managing to avoid potential congestion.

\section{FinAl ReMARKS}

This paper has proposed a SPH method for scheduling the times of recharging of EVs. The reported results show that the 
proposed approach can construct recharging schedules that will minimize the impact of voltage drops, and can be easily implemented as a voltage congestion aware frequency control service. Furthermore, the proposed framework could be easily extended to real time and on-line scenarios, since unexpected changes in demand are seen by the particle system as new contained boundaries, in which a particle can easily re-adjust to a new position.

The encouraging results obtained so far warrant further research effort into this type of algorithm; theoretical as well as practical aspects of the proposed approach are worth further investigation.

\section{APPENDIX}

Voltage Drop For SCHEdUle 1 AND SCHEDUle 2

Without loss of generality, the expressions for currents drawn and associated voltage drop $\Delta V_{0,2}$ due to both Schedule 1 and Schedule 2 can be written as follows:

Schedule 1:

$$
\begin{aligned}
& I_{1}(t)=\left(u(t)-u\left(t-t_{1}\right)\right) e^{j \alpha_{1}} \\
& I_{2}(t)=\left(u\left(t-t_{1}\right)-u\left(t-t_{2}\right)\right) e^{j \alpha_{2}}
\end{aligned}
$$

where $u(t)$ is the unit step function $[u(t)=0$ if $t<0$; and $u(t)=1$ otherwise], hence

$$
\begin{aligned}
& \Delta V_{0,2}(t)=\left(u(t)-u\left(t-t_{1}\right)\right) Z_{a} e^{j \alpha_{1}}+ \\
& \quad\left(u\left(t-t_{1}\right)-u\left(t-t_{2}\right)\right)\left(Z_{a}+Z_{b}\right) e^{j \alpha_{2}} .
\end{aligned}
$$

Differentiating (18) with respect to $t$

$$
\begin{aligned}
\frac{\partial \Delta V_{0,2}(t)}{\partial t}=( & \left.\delta(t)-\delta\left(t-t_{1}\right)\right) Z_{a} e^{j \alpha_{1}}+ \\
& \left(\delta\left(t-t_{1}\right)-\delta\left(t-t_{2}\right)\right)\left(Z_{a}+Z_{b}\right) e^{j \alpha_{2}}
\end{aligned}
$$

Therefore, $\partial \Delta V_{0,2}(t) / \partial t=0$ over the open intervals $\left(0, t_{1}\right)$ and $\left(t_{1}, t_{2}\right)$, and $\partial \Delta V_{0,2}(t) / \partial t \neq 0$ for $t=t_{1}$. To calculate the maximum value of $\left|\Delta V_{0,2}(t)\right|_{1}$, the drop in both interval $\left(0, t_{1}\right)$ and in interval $\left(t_{1}, t_{2}\right)$ need to be considered:

$$
\left|\Delta V_{0,2}(t)\right|_{1}=\left|Z_{a}\right|_{1} \text { for } t \in\left(0, t_{1}\right)
$$

and

$$
\left|\Delta V_{0,2}(t)\right|_{1}=\left|Z_{a}+Z_{b}\right| \text { for } t \in\left(t_{1}, t_{2}\right)
$$

Let $Z_{a}=\left|Z_{a}\right| \angle \gamma_{a}$, and $Z_{b}=\left|Z_{b}\right| \angle \gamma_{b}$. Since the resistance of secondary circuit cannot be negative or zero and secondary circuits are inductive

$$
\left|\gamma_{a}-\gamma_{b}\right|<\frac{\pi}{2} \operatorname{rad}
$$

Now, for any two complex numbers $Z_{a}$ and $Z_{b}$

$$
\left|Z_{a}+Z_{b}\right|^{2}=\left|Z_{a}\right|^{2}+\left|Z_{b}\right|^{2}+2\left|Z_{a}\right|\left|Z_{b}\right| \cos \left(\gamma_{a}-\gamma_{b}\right)
$$

From (23) and using (22), $\left|Z_{a}+Z_{b}\right|>\left|Z_{a}\right|$, hence

$$
\max \left(\left|\Delta V_{0,2}(t)\right|_{1}\right)=\left|Z_{a}+Z_{b}\right| \text {. }
$$

Schedule 2: In Schedule 2, relative to Schedule 1, the magnitude of currents can be halved and duration of currents can be doubled. Thus

$$
\begin{aligned}
& I_{1}(t)=\frac{1}{2}\left(u(t)-u\left(t-t_{2}\right)\right) e^{j \alpha_{1}} \\
& I_{2}(t)=\frac{1}{2}\left(u(t)-u\left(t-t_{2}\right)\right) e^{j \alpha_{2}}
\end{aligned}
$$

hence

$$
\begin{aligned}
& \Delta V_{0,2}(t)=\frac{1}{2}\left(u(t)-u\left(t-t_{2}\right)\right) Z_{a} e^{j \alpha_{1}}+ \\
& \frac{1}{2}\left(u(t)-u\left(t-t_{2}\right)\right)\left(Z_{a}+Z_{b}\right) e^{j \alpha_{2}} .
\end{aligned}
$$

Differentiating (27) with respect to $t$

$$
\begin{aligned}
\frac{\partial \Delta V_{0,2}(t)}{\partial t}= & \frac{1}{2}\left(\delta(t)-\delta\left(t-t_{2}\right)\right) Z_{a} e^{j \alpha_{1}}+ \\
& \frac{1}{2}\left(\delta(t)-\delta\left(t-t_{2}\right)\right)\left(Z_{a}+Z_{b}\right) e^{j \alpha_{2}}
\end{aligned}
$$

Therefore, $\partial \Delta V_{0,2}(t) / \partial t=0$ over the open interval $\left(0, t_{2}\right)$. To calculate the maximum value of $\left|\Delta V_{0,2}(t)\right|$, the drop in the interval $\left(0, t_{2}\right)$ is considered:

$$
\max \left(\left|\Delta V_{0,2}(t)\right|_{2}\right)=\left|\frac{1}{2}\left(Z_{a} e^{j \alpha_{1}}\right)+\frac{1}{2}\left(Z_{a}+Z_{b}\right) e^{j \alpha_{2}}\right| .
$$

Using the triangular inequality

$$
\max \left(\left|\Delta V_{0,2}(t)\right|_{2}\right) \leq\left|\frac{1}{2}\left(Z_{a}\right)\right|+\left|\frac{1}{2}\left(Z_{a}+Z_{b}\right)\right|
$$

and using the fact that (22), $\left|Z_{a}+Z_{b}\right|>\left|Z_{a}\right|$

$$
\max \left(\left|\Delta V_{0,2}(t)\right|_{2}\right)<\left|\frac{1}{2}\left(Z_{a}+Z_{b}\right)\right|+\left|\frac{1}{2}\left(Z_{a}+Z_{b}\right)\right|
$$

and hence

$$
\max \left(\left|\Delta V_{0,2}(t)\right|_{2}\right)<\left|\left(Z_{a}+Z_{b}\right)\right|
$$

Note that the right-hand side of (32) is the same as the right-hand side of (24), therefore

$$
\max \left|\Delta V_{0,2}(t)\right|_{2}<\max \left|\Delta V_{0,2}(t)\right|_{1} .
$$

\section{ACKNOWLEDGMENT}

The authors would like to thank the anonymous reviewers for their valuable feedback on the previous versions of this paper.

\section{REFERENCES}

[1] P. Moses, M. A. S. Masoum, and S. Hajforoosh, "Overloading of distribution transformers in smart grid due to uncoordinated charging of plug-in electric vehicles," in Proc. 2012 IEEE PES Innovative Smart Grid Technologies (ISGT), 2012, pp. 1-6.

[2] P. Richardson, D. Flynn, and A. Keane, "Local versus centralized charging strategies for electric vehicles in low voltage distribution systems," IEEE Trans. Smart Grid, vol. 3, no. 2, pp. 1020-1028, 2012. 
[3] Q. Hamid and J. Barria, "Distributed recharging rate control for energy demand management of electric vehicles," IEEE Trans. Power Syst., vol. 28, no. 3, pp. 2688-2699, Aug. 2013.

[4] P. Zhang, K. Qian, C. Zhou, B. Stewart, and D. Hepburn, "A methodology for optimization of power systems demand due to electric vehicle charging load," IEEE Trans. Power Syst., vol. 27, no. 3, pp. 1628-1636, Aug. 2012.

[5] K. Valentine, W. G. Temple, and K. M. Zhang, "Intelligent electric vehicle charging: Rethinking the valley-fill," J. Power Sources, vol. 196, no. 24, pp. 10717-10726, 2011.

[6] W. Kempton and J. Tomic, "Vehicle-to-grid power implementation: From stabilizing the grid to supporting large-scale renewable energy," J. Power Sources, vol. 144, no. 1, pp. 280-294, 2005.

[7] C. D. White and K. M. Zhang, "Using vehicle-to-grid technology for frequency regulation and peak-load reduction," J. Power Sources, vol. 196, no. 8, pp. 3972-3980, 2011.

[8] J. Taylor, A. Maitra, M. Alexander, D. Brooks, and M. Duvall, "Evaluations of plug-in electric vehicle distribution system impacts," in Proc. 2010 IEEE Power and Energy Soc. General Meeting, 2010, pp. 1-6.

[9] K. Clement, E. Haesen, and J. Driesen, "Coordinated charging of multiple plug-in hybrid electric vehicles in residential distribution grids," in Proc. IEEE/PES Power Syst. Conf. Expo., 2009 (PSCE '09), Mar. 2009, pp. 1-7.

[10] P. Rezaei, J. Frolik, and P. D. Hines, "Packetized plug-in electric vehicle charge management," IEEE Trans. Smart Grid, vol. 5, no. 2, pp. 642-650, 2014.

[11] E. Sortomme, M. M. Hindi, S. J. MacPherson, and S. Venkata, "Coordinated charging of plug-in hybrid electric vehicles to minimize distribution system losses," IEEE Trans. Smart Grid, vol. 2, no. 1, pp. 198-205, 2011.

[12] S. Deilami, A. S. Masoum, P. S. Moses, and M. A. Masoum, "Realtime coordination of plug-in electric vehicle charging in smart grids to minimize power losses and improve voltage profile," IEEE Trans. Smart Grid, vol. 2, no. 3, pp. 456-467, 2011.

[13] K. Clement-Nyns, E. Haesen, and J. Driesen, "The impact of charging plug-in hybrid electric vehicles on a residential distribution grid," IEEE Trans. Power Syst., vol. 25, no. 1, pp. 371-380, Feb. 2010.

[14] M. Gustafson, J. Baylor, and S. Mulnix, "The equivalent hours loss factor revisited power systems," IEEE Trans. Power Syst., vol. 3, no. 4, pp. 1502-1508, Nov. 1988.

[15] O. M. Mikic, "Variance-based energy loss computation in low voltage distribution networks," IEEE Trans. Power Syst., vol. 22, no. 1, pp. 179-187, Feb. 2007.

[16] R. Taleski and D. Rajicic, "Energy summation method for energy loss computation in radial distribution networks," IEEE Trans. Power Syst., vol. 11, no. 2, pp. 1104-1111, May 1996.

[17] A. Masoum, S. Deilami, P. Moses, M. Masoum, and A. Abu-Siada, "Smart load management of plug-in electric vehicles in distribution and residential networks with charging stations for peak shaving and loss minimisation considering voltage regulation," IET Gener., Transm., Distrib., vol. 5, no. 8, pp. 877-888, 2011.

[18] J. J. Monaghan, "Smoothed particle hydrodynamics progress," Rep. Progr. Phys., vol. 68, no. 8, p. 1703, 2005.

[19] M. Müller, D. Charypar, and M. Gross, "Particle-based fluid simulation for interactive applications," in Proc. 2003 ACM SIGGRAPH/Eurographics Symp. Comput. Animation, ser. SCA '03. Switzerland: Eurographics Association, Aire-la-Ville, Switzerland, 2003, pp. 154-159.

[20] J. Short, D. Infield, and L. Freris, "Stabilization of grid frequency through dynamic demand control," IEEE Trans. Power Syst., vol. 22, no. 3, pp. 1284-1293, Aug. 2007.
[21] P. Palensky and D. Dietrich, "Demand side management: Demand response, intelligent energy systems, and smart loads," IEEE Trans. Ind. Informat., vol. 7, no. 3, pp. 381-388, 2011.

[22] D. Angeli and P.-A. Kountouriotis, "A stochastic approach to 'dynamic-demand' refrigerator control," IEEE Trans. Control Syst. Technol., vol. 20, no. 3, pp. 581-592, 2012.

[23] J. Lee, O. Nam, and B. Cho, "Li-ion battery $\{\mathrm{SOC}\}$ estimation method based on the reduced order extended Kalman filtering hybrid electric vehicles," J. Power Sources, vol. 174, no. 1, pp. 9-15, 2007.

[24] S. Lee, J. Kim, J. Lee, and B. Cho, "State-of-charge and capacity estimation of lithium-ion battery using a new open-circuit voltage versus state-of-charge," J. Power Sources, vol. 185, no. 2, pp. 1367-1373, 2008.

[25] V. Gungor, D. Sahin, T. Kocak, S. Ergut, C. Buccella, C. Cecati, and G. Hancke, "Smart grid technologies: Communication technologies and standards," IEEE Trans. Ind. Informat., vol. 7, no. 4, pp. 529-539, 2011.

[26] T. Ikeya, N. Sawada, J. I. Murakami, K. Kobayashi, M. Hattori, N. Murotani, S. Ujiie, K. Kajiyama, H. Nasu, H. Narisoko, Y. Tomaki, K. Adachi, Y. Mita, and K. Ishihara, "Multi-step constant-current charging method for an electric vehicle nickel/metal hydride battery with high-energy efficiency and long cycle life," J. Power Sources, vol. 105 , no. 1, pp. 6-12, 2002.

[27] Y.-H. Liu and Y.-F. Luo, "Search for an optimal rapid-charging pattern for li-ion batteries using the Taguchi approach," IEEE Trans. Ind. Electron., vol. 57, no. 12, pp. 3963-3971, Dec. 2010.

[28] P. Anderson et al., Power System Protection. New York, NY, USA: Wiley, 1998.

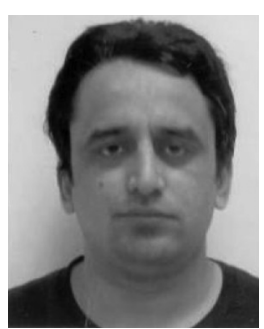

Qazi R. Hamid received the B.E. degree in electrical engineering from the University of Engineering and Technology Peshawar, Pakistan, in 2004 and the M.Sc. and Ph.D. degrees from Imperial College London, London, U.K., in 2007 and 2014, respectively.

His research interests include multi-agent systems, distributed dynamic resource allocation, power system operations, management of smart grids, and subsea power distribution.

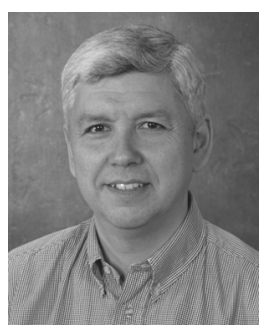

Javier A. Barria (M'02) received the Ph.D. degree in electrical and electronic engineering and the M.B.A. degree from the Imperial College London, London, U.K., in 1994 and 1997, respectively.

$\mathrm{He}$ is currently a Reader with the Intelligent Systems and Networks Group, Department of Electrical and Electronic Engineering, Imperial College London. He has been the joint holder of several European Union Framework and U.K. Engineering and Physical Sciences Research Council project contracts, which are all concerned with aspects of communication systems design and management. His research interests include monitoring strategies for communication and transport networks and distributed resource allocation in dynamic topology networks.

Dr. Barria is a Fellow of the Institution of Engineering and Technology and a Chartered Engineer in the U.K. He was a British Telecom Research Fellow from 2001 to 2002 . 